\title{
Le religieux et l'école en France
}

Table ronde

Religion in schools in France.

La religión en la escuela en Francia

André Blandin, André Legrand, Philippe Le Guillou, Mark Sherringham et Jean-Paul Willaime

\section{OpenEdition Journals}

Édition électronique

URL : http://journals.openedition.org/ries/1453

DOI : 10.4000/ries.1453

ISSN : 2261-4265

Éditeur

Centre international d'études pédagogiques

Édition imprimée

Date de publication : 1 septembre 2004

Pagination : 49-70

ISBN : 978-2-85420-561-8

ISSN : $1254-4590$

Référence électronique

André Blandin, André Legrand, Philippe Le Guillou, Mark Sherringham et Jean-Paul Willaime, « Le religieux et l'école en France », Revue internationale d'éducation de Sèvres [En ligne], 36 | septembre 2004, mis en ligne le 18 novembre 2011, consulté le 19 avril 2019. URL : http:// journals.openedition.org/ries/1453; DOI : 10.4000/ries.1453 


\section{Le religieux et l'école en France}

\section{Table ronde ${ }^{1}$}

\section{Pour ouvrir le débat}

Dominique Borne: Notre débat porte sur l'ensemble des problèmes juridiques, institutionnels, d'enseignement etc. posés par la présence du religieux à l'école. Nous ne traitons donc pas seulement les problèmes d'enseignement du fait religieux ou les problèmes de la laïcité. Notre approche est globale. Afin de lancer le débat je voudrais présenter quatre propositions introductives.

Première proposition: ces problèmes se situent dans une tension entre l'égalité et la liberté. L'article dix de la Déclaration des droits de l'homme de 1789 précise que «nul ne doit être inquiété pour ses opinions, même religieuses, pourvu que leur manifestation ne trouble pas l'ordre public». Le religieux est ici placé dans le domaine de l'opinion, de la libre opinion. Un siècle plus tard la loi d'orientation de 1989 ne dit pas autre chose: «dans les collèges et les lycées, les élèves disposent, dans le respect du pluralisme, du principe de neutralité, de la liberté d'information et de la liberté d'expression. L'exercice de cette liberté ne peut porter atteinte aux activités d'enseignement». Je retrouve, dit autrement, l'«ordre public» de la Déclaration des droits de l'homme. Les membres de la communauté éducative, professeurs, élèves, etc. peuvent s'exprimer dès lors qu'ils n'enfreignent pas ce que j'appelle l'«égalité citoyenne». L'École - les élèves ne sont certes pas des citoyens - contribue à la construction des citoyens, des citoyens naturellement égaux entre eux, qui obéissent tous aux mêmes lois. En ce sens, peu importe qu'ils professent par ailleurs des opinions, peu importe même que ces opinions soient religieuses. Il faut donc tenir ensemble dans une tension que je crois féconde l'égalité citoyenne et la liberté d'expression. C'est ainsi qu'il faut appréhender les problèmes du religieux à l'école.

Deuxième proposition: les manifestations actuelles du religieux à l'école relèvent davantage du communautarisme ou d'affirmations multiples

\footnotetext{
1. Table ronde organisée au CIEP le 29 avril 2004. Elle a réuni : André Blandin, secrétaire général adjoint de l'Enseignement catholique ; Dominique Borne, doyen de l'Inspection générale de l'Éducation nationale ; André Legrand, professeur de droit public à l'université de Paris X Nanterre ; Philippe Le Guillou, inspecteur général de lettres, Mark Sherringham, directeur de l'IUFM d'Alsace; Jean-Paul Willaime, directeur d'études, directeur du groupe de sociologie des religions et de la laïcité, IRESCO, École Pratique des Hautes Études.

Transcription : Marie-José Sanselme.
} 
d'identité. Ces manifestations prennent les couleurs du religieux, s'habillent des oripeaux du religieux, on peut douter qu'elles soient fondamentalement religieuses. Nous ne sommes dans la situation de la France avant la loi de séparation de l'Église et de l'État de 1905. Ce n'est pas ce type de conflit. Est-il certain que le turban des Sikhs soit un signe religieux?

Troisième proposition: aborder le religieux en se référant sans cesse à la laïcité est à mon sens une erreur. Je crois que c'est une erreur d'avoir imposé aux IUFM un module intitulé «Philosophie de la laïcité et enseignement du fait religieux». Je pense qu'il faut être pénétré de la philosophie de la laïcité pour enseigner aussi les mathématiques et pas seulement le fait religieux. L'enseignement du fait religieux relève du domaine des connaissances parmi d'autres connaissances.

Quatrième proposition: dans le domaine qui nous réunit aujourd'hui, la France, qui a longtemps fait figure d'îlot laïque en Europe, est de moins en moins une exception. De plus en plus, les autres pays européens se rapprochent de nous et les écarts se réduisent. Par exemple, en Grèce, la religion figurait sur la carte d'identité jusqu'à une date très récente; c'est fini maintenant. En Suède, il n'y a plus de religion d'État. On pourrait montrer que, progressivement, l'Europe se construit malgré les différences, et que les États européens tous ont en commun l'affirmation de la liberté religieuse, l'égalité des cultes, même quand il existe une religion d'État, et le pluralisme religieux.

\section{Le droit d'expression religieuse entre évolution et continuité}

André Legrand: J'aurais tendance à dire que c'est aussi nous qui nous rapprochons de plus en plus des autres pays européens. La question du foulard islamique est éclairante sur ce point. Il existe une querelle sur le point de savoir si la position exprimée par le Conseil d'État, dans son avis de 1989, a constitué ou non une novation. Je pense qu'elle l'a été en partie parce que, dans son analyse du droit applicable, le Conseil a intégré, à côté des textes constitutionnels et des textes législatifs, toute une série de textes internationaux signés par la France et qui impliquent un certain nombre d'obligations. Ces textes, par exemple la Convention européenne des droits de l'homme ont nécessairement induit une nouvelle lecture de nos principes nationaux. Notre pays manque cruellement de mémoire. Il aurait dû se rappeler que les débats qui l'ont agité en 2003 avaient déjà eu lieu, sous une forme assez similaire, à l'époque où François Bayrou était ministre et où l'on se demandait s'il fallait une loi pour interdire le foulard. Déjà, un ministre y avait renoncé parce qu'il n'était pas certain qu'elle passe la barrière de la censure du Conseil constitutionnel d'abord, puis celle de la Cour européenne des droits de l'homme. Et on n'en est toujours pas certain en ce qui concerne la loi qui vient d'être votée. À cette époque, en 1994-1995, la position de la plupart des membres les plus éminents 
du Conseil d'État était de dire que l'avis du Conseil d'État de 1989 et la jurisprudence Kherouaa s'inscrivaient dans une tradition ancienne. Ils faisaient référence à l'œuvre pacificatrice du Conseil d'État après la loi de séparation de l'Église et de l'État, à travers toute une série de jurisprudences sur les problèmes de sonneries de cloches ou d'interdiction des processions par les maires par exemple. Sur ces questions, le Conseil avait adopté la même attitude qu'actuellement, c'est-à-dire une analyse au cas par cas et non des principes d'interdiction générale et absolue. La thèse de la continuité n'est donc pas infondée. Mais, en même temps, des évolutions importantes se sont aussi produites. Bernard Toulemonde ${ }^{2}$ cite fréquemment la fameuse circulaire de Jean Zay à laquelle on se réfère en permanence: "l'école doit être le lieu sacré où les querelles des hommes ne pénètrent pas». Nous avons donc évolué; notre vision de l'École, du principe de laïcité, de la présence du fait religieux à l'école a changé. Et cela s'inscrit dans une évolution plus large dont le décret de 1991 sur le droit des élèves témoigne aussi. L'une des questions majeures derrière tous ces problèmes, c'est effectivement celle de la tension entre la liberté et l'égalité. À partir des années quatre-vingts, et cela s'est accéléré au cours des années quatre-vingt-dix, on a renoncé, sur le plan juridique, à l'idée de l'école sanctuaire ou de l'école asile. Qu'est-ce qu'un asile? C'est un lieu où le droit de l'extérieur ne s'applique pas et où la compétence juridique est dévolue à d'autres autorités que celles qui règlent l'extérieur. Il fut, c'est vrai, une période où l'école était un asile ou un sanctuaire parce qu'on n'y appliquait pas les règles applicables ailleurs. L'évolution qui s'est produite - et qui touche très directement au problème de l'expression religieuse - a été précisément que, pour les élèves, cette notion d'asile a disparu et que la frontière est tombée. On s'est mis à considérer que les élèves pouvaient entrer à l'école sans abandonner à la porte les droits qu'ils avaient à l'extérieur, droit d'expression religieuse mais aussi tous les droits qui sont reconnus dans les textes récents et qui sont finalement tout aussi contestés que les problèmes d'expression religieuse, parce qu'ils posent également d'importants problèmes concrets au sein des établissements scolaires, le droit d'association, le droit de publication, le droit de réunion. C'est sur ce plan-là que la grande évolution s'est produite. On a admis qu'un élève ne se transformait pas en franchissant les portes de l'école et qu'à condition que cela reste compatible avec le fonctionnement de l'institution scolaire, il pouvait y exercer divers droits qu'il avait à l'extérieur. Certes, ces droits ne sont pas absolus; ils sont un peu plus limités dedans que dehors: on sait, par exemple, la distinction parfois subtile que la juridiction administrative établit entre «réunions politiques» et «réunions d'information». On ne peut s'interroger sur la question du religieux à l'école sans la replacer dans le contexte plus vaste des droits et libertés dont disposent les élèves.

2. Bernard Toulemonde : ancien recteur, inspecteur général de l’Éducation nationale. 


\section{Les établissements privés sous contrat et leur "caractère propre "}

André Blandin: L'Enseignement catholique sous contrat représente actuellement deux millions d'élèves, soit près de $20 \%$ de la population scolaire. Selon les enquêtes de Langouet et Léger, un élève sur deux passe au moins une année de sa scolarité dans l'enseignement sous contrat. C'est dire l'imbrication avec l'enseignement public. Le «zapping» s'accroît pour les raisons les plus diverses. Les sondages manifestent la diminution de la motivation religieuse ou plutôt «exprimée comme religieuse». En fait, la situation est complexe, les motifs sont multiples: du contournement des contraintes de la sectorisation jusqu'au recours en cas d'échec en passant par des choix éducatifs explicites...

Ensuite, l'enseignement catholique est une réalité plurielle. Il n'y a pas d'homogénéité entre des établissements situés en centre-ville et ceux qui sont dans les banlieues, moins nombreux que ceux de l'enseignement public pour des raisons historiques et de moyens. Entre certains établissements de centreville qui pratiquent une catéchèse obligatoire et une école de Marseille qui reçoit $85 \%$ d'élèves musulmans, l'écart est énorme.

L'Enseignement catholique représente en fait 8500 centres de décision puisqu'il est constitué de 8500 établissements. Seuls les établissements sont reconnus par la loi Debré. "Chimère de penser que nous allons édifier, face à l'Éducation nationale, une forteresse (...)»disait, en substance, Michel Debré le 23 décembre 1959 en présentant sa loi au Parlement. Voilà aussi l'une de nos particularités. Cependant, sans pouvoir hiérarchique sur les établissements, les instances diocésaines ou nationales sont dans une situation d'animation qui n'est pas dénuée d'intérêt.

Comment définir le caractère propre $^{3}$ ? Le statut de l'Enseignement catholique, promulgué en 1992, parle de "l'établissement catholique d'enseignement». Ce n'est pas l'enseignement lui-même qui est catholique, c'est l'établissement qui est porteur du caractère propre. La différence est de taille! Dans sa définition actuelle, le caractère propre est le lien entre "enseigner», «éduquer» et "proposer un sens à la vie éclairé par l'Évangile». Nous ne sommes pas dans le registre de l'obligation mais dans celui de l'appel, de la proposition. Les enseignants sont tenus de respecter le projet éducatif de l'établissement, mais ils n'ont pas obligation de le mettre en œuvre: un certain nombre d'enseignants ne sont pas de confession catholique. Cela permet de vivre entre adultes le respect de la liberté de conscience due aux enseignants et aux jeunes. Certains sont allés jusqu'à parler d'un "enseignement laïc» à l'intérieur d'un établissement associé à l'État. Je ne pense pas qu'il faille aller jusque-

3. "L'établissement tout en gardant son caractère propre doit donner cet enseignement dans le respect total de la liberté de conscience. Tous les enfants sans distinction d'origine, d'opinion ou de croyances y ont accès. » Loi 59-1557 du 31 décembre 1959, dite Loi Debré, article 1. 
là, mais en tout cas ce n'est pas un «enseignement religieux». L'établissement est porteur du caractère propre: il n'y a pas d'étanchéité entre enseignement et éducation. Le lien est de l'ordre des pratiques pédagogiques. Ainsi, la façon dont un enseignant évalue les résultats de ses élèves dit sa vision des personnes et son projet éducatif. Un système d'évaluation fondé sur la comptabilisation des erreurs et des fautes ou un autre fondé sur la valorisation positive des acquis ne traduit pas le même regard sur le développement de la personne!

Enfin, quand on parle de "proposer un sens à la vie», il s'agit bien d'une proposition et non d'une obligation. Nulle part maintenant, dans les établissements catholiques, la catéchèse explicite ne doit être obligatoire.

Revenons à la différence entre un enseignement confessionnel et un enseignement catholique et donnons un exemple : il y a un enseignement catholique dans des pays de confession musulmane. Au Maroc, des établissements catholiques accueillent $100 \%$ d'élèves musulmans et il s'agit pourtant d'établissements «catholiques» parce qu'ils visent d'abord le développement de l'homme et qu'ils ne sont ni confessionnels ni prosélytes. Voilà le terrain du dialogue possible avec les établissements publics.

Dominique Borne: Vous posez le problème des valeurs. Je retiens cela pour le débat futur. Je retiens aussi ce que disait André Legrand en citant Jean Zay: c'était l'époque, peut-être, où l'école exerçait un sacré de substitution et c'est peut-être ce sacré de substitution qui a disparu.

André Blandin: Cette allusion aux «valeurs» me permet de préciser un peu notre position: il n'y a pas un catalogue chosifiant de valeurs évangéliques opposées à des valeurs humaines. Il y a une exigence évangélique de faire croire en l'humanité. C'est sur ce terrain-là que nous pouvons nous retrouver et nous reconnaître mutuellement.

\section{Sociologie du fait religieux en France}

Jean-Paul Willaime: Nous sommes dans une situation socioreligieuse paradoxale au sens où, si beaucoup moins de Français s'identifient aux religions instituées, en particulier à la principale institution religieuse de notre pays, l'Église catholique, de nombreuses personnes s'intéressent au religieux et à la spiritualité. Par rapport aux dernières décennies, les évolutions sont sensibles. Le taux de pratique cultuelle a fortement baissé depuis l'après-guerre mais il était, ces dernières années, plutôt étale. La relative baisse de l'identification aux formes les plus instituées du religieux contraste avec la forte présence publique, en particulier médiatique, du religieux dans la société actuelle. On observe un intérêt du public pour le domaine religieux dans la variété de ses expressions et aussi, de façon plus directe, pour les questions de spiritualité. Cela se traduit notamment par une augmentation du nombre de Français s'identifiant à des traditions religieuses minoritaires: bien entendu l'islam, mais aussi le boud- 
dhisme et divers mouvements religieux qui, pour la plupart, ne comptent qu'un nombre réduit de fidèles. Cette pluralité religieuse accentuée et très complexe conduit souvent à des approximations et à des amalgames (comme on l'a vu dans le débat public sur les «sectes»). Avant de parler du clivage des générations, il faut souligner qu'il existe en France une large acceptation de la laïcité, notamment scolaire, y compris parmi les Français catholiques pratiquants réguliers comme l'a montré une enquête menée en 1998. L'élément le plus significatif de l'évolution qui nous intéresse particulièrement ici c'est le clivage entre les générations. Toutes les données d'enquête quantitatives nationales et européennes montrent des différences très importantes entre les jeunes adultes et les tranches d'âge plus élevées. Chez les jeunes adultes - les personnes entre dixhuit et vingt-quatre ans -, on constate en France, aux Pays-Bas et en GrandeBretagne, pays où le phénomène est le plus marqué en Europe, que la majorité des nouvelles classes d'âge ne s'identifie plus à une confession religieuse. C'est la conséquence de la baisse de la socialisation religieuse des jeunes, baisse qui n'est pas sans conséquences sur l'école publique. Les enseignants sont aujourd'hui confrontés à des élèves qui, pour la plupart, ne reçoivent pas une formation religieuse en dehors de l'école. On ne mesure peut-être pas encore tout à fait l'ensemble des conséquences sociales et culturelles de cet important changement. Dans les années cinquante/soixante, un grand nombre de Français avaient été formés dans une religion, même s'ils ne la pratiquaient pas ou avaient pris leurs distances par rapport à cette socialisation primaire. Mais ils avaient reçu des éléments de connaissances et des points de repères symboliques et éthiques que seule une minorité de jeunes reçoivent aujourd'hui. De là le problème très débattu et commenté de l'inculture religieuse des jeunes générations, de leur analphabétisme religieux, un phénomène qui a été vérifié à travers divers indicateurs, aussi bien dans les enquêtes quantitatives que qualitatives. Des notions, des vocabulaires, des images, des personnages et en particulier des personnages bibliques ou des expressions comme «il a eu son chemin de croix» ou «l'ouvrier de la dernière heure», etc. ne font plus sens ou sont totalement incomprises des jeunes générations mais aussi, de plus en plus souvent, des moins jeunes. On constate aussi cette inculture religieuse chez un trop grand nombre de journalistes qui, lorsqu'ils ont à traiter un sujet religieux, ne disposent plus du vocabulaire adéquat ou font des bourdes énormes. Cette perte de références culturelles et d'intelligibilité, avec toutes les conséquences qu'elle peut avoir sur l'enseignement de la littérature, des langues, de l'histoire, de la géographie, des arts, etc., concerne également les jeunes professeurs. Je le constate également à l'École Pratique des Hautes Études, au niveau du troisième cycle. Ce n'est donc pas l'apanage de tel ou tel niveau d'enseignement. En même temps, on note des curiosités et des questions naïves chez les jeunes comme chez les moins jeunes, dues aux répercussions de l'actualité nationale et internationales et aux effets du traitement médiatique du religieux. De fait, l'on parle 
surtout du religieux lorsqu'il pose problème, lorsqu'il est lié à des conflits, à des violences, à des fanatismes, etc. Des données d'enquête montrent que, dans l'opinion publique, le religieux est plus associé à l'intolérance et au fanatisme qu'à la tolérance et à la paix. C'est le résultat de la diffusion sociale d'une image médiatique du religieux qui traite de l'actualité, des problèmes, des conflits et des tragédies qui y sont liées. L'image sociale du religieux est aujourd'hui, passablement brouillée et les rapports des Français à la religion sont très ambivalents, entre la fascination et la répulsion, entre l'attirance pour des expériences spirituelles et l'identification à des "héros du croire» d'un côté, la dérision et l'anticléricalisme de l'autre. En sociologie des religions aujourd'hui, nous sommes amenés à faire des distinctions inattendues comme celles entre les " sans religion croyant " et les «sans religion non croyant ». Il y a en effet des personnes qui s'identifient religieusement et dont le niveau de croyance est très faible - ce qui est parfois surprenant par rapport à leur identification nominale à une tradition religieuse - et des personnes se déclarant sans religion qui font état de croyances. Les enquêtes européennes sur les valeurs ont en particulier constaté une remontée de la croyance dans l'«après mort» particulièrement chez les jeunes. C'est un effet de la dérégulation du religieux et on peut dire, reprenant la notion durkheimienne d'anomie, qu'il existe aujourd'hui une certaine anomie religieuse. Une autre donnée d'enquête intéressante pour tous les éducateurs est la suivante : les catégories de Français qui s'avèrent le plus imperméables aux sciences occultes, au paranormal, à l'ésotérisme, à l'astrologie, aux tables tournantes, etc., sont les «catholiques pratiquants réguliers » et les " athées convaincus ». Le point commun de ces Français est qu'ils sont inscrits dans un système de conviction, qu'il soit de l'ordre de l'athéisme ou de l'ordre d'une affirmation religieuse. Par contre, les Français les plus perméables aux sciences occultes et au paranormal, ce sont ceux les catholique nominaux, les non pratiquants (je n'évoque pas ici les minorités car il s'agit de référence à des données quantitatives). Selon certaines théories de la sécularisation, plus les sociétés devenaient modernes, moins elles étaient religieuses. Ces théories sont remises en cause aujourd'hui : la modernité, ce n'est pas moins de religieux, c'est du religieux autrement, du religieux dérégulé, atomisé. Ce qui est clair, d'un point de vue sociologique, c'est qu'il y a eu une baisse effective du pouvoir social d'encadrement et d'imposition des grandes institutions religieuses. C'est sur le fond de cet affaiblissement de l'encadrement social et culturel du religieux par les institutions que l'on assiste à l'efflorescence et la dissémination d'un sentiment religieux sauvage, dérégulé. Situation propice aux petits entrepreneurs dans le domaine du salut qui peuvent susciter de l'intérêt s'ils sont suffisamment habiles pour développer un message religieux associant bien-être physique et psychique, un message devenant une formule de bien-être. Ce qui nous rappelle que la religion a quelque chose à voir avec la gestion symbolique du bonheur et du malheur. 
Dominique Borne: Nous constatons tous que la sécularisation de la société va de pair avec la floraison de multiples formes du religieux. Les grands appareils - on disait autrefois idéologiques - qui contrôlaient l'ensemble social, perdent du terrain. Le religieux est plus dispersé et simultanément on assiste à une perte globale de sens. Nous avons perdu deux éléments majeurs de l'ancien monde : le rural et le religieux. Les élèves ne savent plus comment on laboure avec une charrue, comment on attelle les bœufs; ils ne savent pas plus le sens du plan d'une église. Le monde rural s'enfuit avec le monde religieux.

\section{Le fait religieux en classe}

Philippe Le Guillou: Dans certaines classes aujourd'hui, dans des disciplines comme les lettres ou l'histoire, il arrive que des élèves contestent l'authenticité des faits et les interprétations qui sont données. Certains, même, refusent des œuvres parce qu'elles convoient des connotations religieuses qui ne sont pas les leurs et qu'ils contestent en tant que telles. À ce moment-là, c'est l'expression d'appartenance à une communauté qui l'emporte sans doute plus que l'affirmation d'une religion, si tant est qu'il y ait même une croyance religieuse réelle dans ces réactions. L'autre fait qu'observent en particulier les inspecteurs généraux qui ont à présider des concours de recrutement de nouveaux professeurs, c'est cet analphabétisme religieux qui fait que, de plus en plus, des pans entiers de notre culture sont peu lisibles pour un certain nombre de candidats à ces fonctions, alors même qu'ils auront à les transmettre en tant que professeurs. Nous sommes bien dans le domaine des connaissances. Lorsque les nouveaux programmes de lettres ont été mis en œuvre à partir de septembre 1996 pour les classes de collège, on a parlé de l'enseignement des textes dits fondateurs. Dans ces textes qui permettent de rapprocher la perspective historique, la perspective littéraire et d'autres perspectives, ce qui était important, c'était de réaffirmer ce socle gréco-latin qui fait que des élèves de sixième ont aujourd'hui une approche du phénomène religieux - ou du fait religieux ou du religieux - à travers la lecture et la fréquentation d'extraits de l'Énéide, de l'Odyssée et de la Bible sans qu'il y ait, et c'est très important, une prééminence d'un texte par rapport aux autres. On est dans une perspective polyphonique, plurielle. On observe des textes, des faits, des phénomènes culturels sans leur dénier toute perspective d'ordre catéchistique ou apologétique, mais ce n'est pas sur ces points que l'on met prioritairement l'accent. La Bible, en classe de sixième, apparaît comme un texte fondateur parmi d'autres. L'exigence de pluralité est parfaitement respectée et il est question, dans les programmes, aussi bien du Dieu des chrétiens que des dieux de l'Olympe. Il s'agit plutôt d'esquisser avec les élèves une réflexion sur les relations que l'on peut avoir avec le sacré tout en reconnaissant, bien entendu, que le sacré a, au cours de l'histoire, des expressions et des accomplissements extrêmement variés. Ce qui réunit toutes les disciplines, lorsqu'elles sont en relation avec le reli- 
gieux, c'est de montrer que ce qui nous intéresse, c'est une approche d'ordre anthropologique, qui interroge, met à distance, élit comme matériau de travail les faits, les périodes, les textes et les œuvres. En lettres, à chaque jalon de son cursus de la sixième à la terminale, un élève rencontre aujourd'hui ce que l'on appelle les grands mythes, qui ont de toute évidence une portée et des résonances religieuses. Un aspect important de notre enseignement est l'étude de l'argumentation. En classe de seconde, cela amène à étudier l'argumentation indirecte et la parabole trouve à ce moment là tout naturellement sa place. Ce socle des textes fondateurs permet aux élèves de savoir ce que sont les fêtes religieuses, les cultes, les divinités, l'organisation du temps à travers toutes ces civilisations... Donc, on est - les programmes le disent clairement - dans un domaine de transmission des connaissances et non de prosélytisme ou de conversion. On observe et on transmet des éléments consubstantiels à notre culture. Il va de soi que l'on ne peut pas nier l'existence du religieux faute de se priver de comprendre toute une part de notre culture. Il est difficile, par exemple, de lire La ballade des pendus si l'on ne renvoie pas à la notion de dogme, de communion des saints. Comment comprendre le mythe de Don Juan si on ne le replace pas dans un certain contexte? De même, comment lire un poème de Baudelaire si l'on n'a plus du tout conscience de ce qu'est un encensoir ou un ostensoir? Comment, encore, comprendre la portée du geste de Julien Sorel lorsqu'il tire sur Madame de Rênal si l'on ignore ce qu'est l'élévation? Ce travail d'élucidation d'informations est mené par les professeurs. Il vaut de la même manière pour la lecture du patrimoine. Comment lire un bas relief, un retable, un élément d'un monument si l'on n'a pas ce substrat d'ordre religieux? On peut aussi développer chez les élèves, en relation avec cette question du religieux à l'école, la pensée symbolique d'un côté et l'esprit critique de l'autre. La pensée symbolique nous aide à construire un sens et cette démarche a très souvent partie liée avec ce que l'on appelle l'herméneutique. Là encore, dans le travail sur les textes, sur les objets, sur les faits, on peut mener une lecture de cet ordre. Et en ce qui concerne l'esprit critique, il ne s'agit pas de transmettre des faits religieux en demandant à tel ou tel de s'inféoder à telle ou telle vision du monde. Il s'agit au contraire de montrer qu'il y a une pluralité de ces visions du monde et que, si l'on ne maîtrise pas ces référents bibliques, ces référents d'ordre religieux, on se prive, on s'ampute d'un certain nombre d'éléments essentiels pour la lecture du monde qui nous entoure et de l'histoire à laquelle nous appartenons. Mais il ne faut pas négliger non plus la tradition de l'argumentation, de l'esprit critique qui est une mise à distance essentielle. On peut certes regarder les sources religieuses des textes mais relire aussi Voltaire, certains articles du Dictionnaire philosophique qui interrogent le religieux dans une perspective de mise à distance, d'éveil à une certaine forme de liberté. On retrouve la tension qu'évoquait tout à l'heure Dominique Borne, ce cheminement vers la liberté. Savoir lire et décrypter les faits d'une culture via le religieux, c’est précisément aller vers cette liberté. 
Dominique Borne: N'y a-t-il pas un risque de faire du religieux quelque chose de mort, de l'ordre du patrimoine? C'est ce monde que nous avons perdu, ce monde d'avant qui n'est plus le monde d'aujourd'hui. C'est un vrai problème d'enseignement.

\section{Laïcité et connaissance du fait religieux dans la formation des enseignants}

Mark Sherringham: Le lien entre la formation des enseignants et la laïcité républicaine constitue un cadre à partir duquel on peut mieux saisir la prise en compte du religieux par l'école française. Depuis 1991, date de la création des instituts universitaires de formation des maîtres (IUFM), on peut distinguer deux périodes. La première correspond aux dix premières années d'existence des IUFM, et se fonde sur les circulaires du 2 juillet 1991 et du 14 novembre 1994. Le premier de ces textes introduit une distinction fameuse, même si elle n'est pas nécessairement heureuse, entre la formation disciplinaire et la formation générale. Cette dernière vise " à rendre les futurs enseignants capables de s'adapter aux différents publics scolaires qu'ils sont amenés à rencontrer». C'est souvent dans ce contexte là que les jeunes enseignants sont intéressés par le religieux: comment faire face à un public scolaire qui vient d'une culture ou d'une religion différente de la mienne? Et si moi-même je n'en ai pas, ou plus, comment faire? Le texte officiel ne répond pas à ce type de questions, mais précise que le futur enseignant doit apprendre à se situer par rapport aux finalités de l'éducation et aux objectifs du système éducatif. La circulaire de 1994 indique que les domaines de formation autres que disciplinaires doivent permettre aux professeurs des écoles d'acquérir « une information suffisante sur les caractéristiques du système éducatif, ses valeurs de référence, ses missions, ses structures et sa réglementation». Quant aux professeurs du second degré, il est précisé que le plan de formation doit leur donner l'occasion de «situer la place de l'enseignement secondaire dans l'ensemble du système éducatif français, d'en comprendre les missions essentielles et identifier les valeurs de référence qui fondent sa responsabilité ». C'est à peu près tout ce qu'on trouve dans les textes pendant ces dix années. On remarque d'abord une très grande prudence, voire une certaine frilosité. Ensuite, s'il est fait régulièrement référence aux finalités de l'éducation, et aux valeurs du système éducatif, à aucun moment celles-ci ne sont énoncées ni explicitées. Et, troisièmement, toute mention d'une formation proprement philosophique pour les enseignants disparaît. Les termes "laïcité » et "philosophie» avaient été jusqu'à la création des IUFM des concepts de référence explicites de la formation des maîtres dans notre pays. Avec leur disparition des circulaires ministérielles, tout se passe comme si l'on entrait dans une phase de laïcité implicite voire honteuse - si je voulais être polémique. Le début de l'année 2002 marque un tournant. Le 
rapport Debray sur l'enseignement du fait religieux dans l'école laïque paraît en février. Une circulaire en date du 22 mars 2002 est publiée sur «les principes et organisation de la deuxième année de formation des enseignants stagiaires ». Ce texte national de cadrage, qui est toujours valable aujourd'hui, énonce quatre priorités à la formation en deuxième année d'IUFM, dont l'une consiste à «former un fonctionnaire du service public d'éducation». Dans cette perspective, il convient d'aborder plus particulièrement l'histoire et l'actualité des valeurs de l'École républicaine, des éléments de philosophie et de sociologie de l'éducation, les enjeux de l'école d'aujourd'hui et ses valeurs, l'histoire et l'actualité de la laïcité en relation notamment avec l'enseignement du fait religieux. Pour les professeurs des écoles, il est indiqué que trente heures sur un total de quatre cent cinquante seront consacrées à la connaissance de l'école et de ses valeurs de la manière suivante: éléments de philosophie; déontologie du métier du service public; philosophie de la laïcité; histoire du fait religieux; responsabilité morale, civile et pénale de l'enseignant. Pour les professeurs du second degré, il est précisé que soixante-dix heures au minimum sur deux cent seize seront consacrées à des passages obligés spécifiques tels que: l'analyse des pratiques professionnelles, la déontologie du métier du service public, la philosophie de la laïcité, l'histoire du fait religieux et ensuite la maîtrise des outils de l'enseignement puis l'adaptation et l'intégration scolaire. On voit donc réapparaître deux termes qui avaient complètement disparu: la notion de laïcité et celle de philosophie. Il existe un lien très fort, dans notre enseignement et pas seulement dans la formation des maîtres, entre laïcité et philosophie. L'École française est une École laïque, et c'est aussi une École qui s'achève par une classe de philosophie en terminale. Mais ce qui est nouveau, c'est de rapprocher la laïcité et la philosophie d'une part avec la religion d'autre part: c'est une nouvelle configuration conceptuelle par rapport à la formation des maîtres et c'est en même temps un changement de cap. Réaffirmation explicite de la laïcité comme valeur, référence à la philosophie, et introduction de la religion dans ce contexte, on voit bien que notre École se recentre sur une mission historique, une identité propre. C'est une École laïque respectant la liberté de conscience des élèves, une École qui donne sa place à la philosophie et à la formation du jugement critique de l'élève et c'est une École qui doit être capable de faire de la religion un objet de réflexion et de connaissance dans le respect des croyances de chacun. Le défi, maintenant, est de réussir à réaffirmer explicitement l'identité propre du service public laïc d'éducation et d'instruction en évitant que cette démarche n'aboutisse à la nostalgie d'un passé qui n'a plus lieu d'être ou ne sombre dans la tentation du renfermement sur soi. L'École sanctuaire ne semble pas une finalité intéressante dans le contexte actuel. Pour que la laïcité scolaire française puisse continuer de jouer un rôle d'unité nationale, elle doit approfondir, dans la formation initiale et continue de ses maîtres et dans l'enseignement dispensé, la réflexion proprement philosophique sur ses fon- 
dements conceptuels en acceptant de prendre en compte l'existence du fait religieux et de se confronter à la question du sens à laquelle s'adosse nécessairement toute conviction religieuse. Dans un ouvrage intitulé À l'ombre des lumières, Régis Debray assignait au fait religieux le statut de l'illusion transcendantale chez Kant. En tant qu'elle reste une illusion, si je lis bien Régis Debray, la religion est donc toujours pensée comme ne relevant pas d'une logique de la vérité mais d'un processus de résistance à la vérité. Mais en tant qu'elle est transcendantale, cette illusion est indépassable et structurellement liée à l'usage de notre pensée. La religion serait donc une erreur nécessaire qu'il importe de connaître puisqu'il n'est plus question de la faire disparaître, comme l'espèrent encore les tenants d'une certaine conception plus positiviste de la laïcité. Il me semble que cette position doive à son tour être interrogée. La laïcité ne sera capable d'une refondation et d'un renouvellement que si elle accepte de penser à nouveau la relation de la vérité et des religions et de considérer que la religion n'est pas seulement un problème culturel, mais bien une voie d'accès à la question des finalités ultimes de l'humanité. J'espère que notre École sera en mesure de relever ce défi sur le plan intellectuel ainsi que dans le cadre de la pratique quotidienne de la classe.

\section{Que dire à l'école? Comment le dire?}

Dominique Borne: Je m'interroge sur le sens de l'expression «l'École et ses valeurs». Les valeurs de l'École sont-elles distinctes des valeurs républicaines? Ce sont les valeurs de la République qui, naturellement, sont le soubassement de tout enseignement. Pour certains, l'introduction du fait religieux dans l'enseignement servirait à "ré-enchanter le monde»: ce monde sécularisé, vide, on l'animerait avec des mythes, des paraboles, des symboles. Et puis quelques bonnes valeurs et le sens du péché... On voit le danger de la démarche. L'enseignement du fait religieux n'est pas fait pour ré-enchanter les salles de classe. Dans son dernier livre Ce que voile le voile, Régis Debray regrette la sacralité républicaine de l'École. Quand le religieux disparaît de l'horizon social l'École doit-elle procurer un sacré de substitution?

André Legrand: La tendance actuelle vers une dérégulation religieuse soulève effectivement la question du rôle de l'École. Comment rétablir la cohérence sans tomber du même coup dans le prosélytisme et la conversion? On touche là un aspect essentiel de la question de la confrontation entre l'élève entrant à l'école avec ses convictions et l'institution scolaire ayant un certain nombre d'exigences à respecter.

André Blandin: M. Willaime montrait comment la religion ne structure plus la société : le repli narcissique des élèves sur eux-mêmes est l'une des caractéristiques de notre temps. Dans son livre Catholicisme, la fin d'un monde, Danièle Hervieu-Léger montre bien à la fois la fin des structures ecclésiales et 
une autre perception des rapports entre la société et l'individu. Le temps des idéologies est mort, les grands projets sociétaux a ont du mal et on se centre désormais sur l'accomplissement de chacun. J'ai été frappé aussi par ce qui a été dit sur la simultanéité entre la disparition du monde rural et du monde religieux. Les formes de la religion étaient liées au monde rural et l'un des défis de l'Église en France, pas encore complètement relevé, a été aussi de faire ce passage de la civilisation rurale à la civilisation urbaine.

Dominique Borne: Nous abordons une nouvelle étape de notre débat: comment le religieux se manifeste-t-il à l'École? Que veulent réellement dire les élèves quand ils manifestent ostensiblement? Est-ce vraiment du religieux?

Jean-Paul Willaime: C'est le problème plus général de l'autorité des maîtres et de la reconnaissance de leur légitimité à parler, à instruire sur un certain nombre de questions. Je pense à ces réactions négatives d'élèves qui, au nom de leur opinion, réagissent aux propos d'un enseignant qui parle d'un texte biblique ou d'une sourate coranique en apportant quelques éléments d'histoire sur la constitution et la réception de ces textes. C'est un énorme problème qui s’inscrit dans une situation socioculturelle plus générale. Récemment, dans un débat organisé par les universités de Lyon, l'école normale supérieure et l'antenne lyonnaise de l'IESR, certaines personnes dans le public ont opposé leur opinion à un propos tout à fait fondé concernant un point d'histoire de l'islam tenu par un de mes collègues, sur le mode " c'est votre opinion, ce n'est pas la mienne ». Il s'agit d'un brouillage du statut épistémologique du savoir. Un maître, dans sa fonction d'enseignant, n'exprime pas son opinion personnelle. Il parle à travers une formation, un savoir et il a une certaine légitimité pour le faire.

Dominique Borne: Ce que vous dites est important. Dans les lieux les plus difficiles, les professeurs qui ont la maîtrise intellectuelle de ce dont ils traitent n'ont jamais de problèmes. Les difficultés surviennent lorsqu'on commence à concéder, à mélanger, à ne pas comprendre qu'il y a différents régimes de vérité. Il manque une formation sur la vérité dans les IUFM. Dire «c'est faux, c'est vrai », en raison, par rapport à la religion n'a pas de sens. Le religieux n'est pas faux ou vrai. Il relève d'un autre régime de vérité. Autre exemple de concession: récemment, un professeur d'histoire avait projeté la visite d'une église. Des élèves musulmans annoncent leur refus d'y entrer. On fait la bêtise - pardonnez-moi - de consulter un imam. L'École n'a pas à consulter un imam. Si conduire des élèves dans une église fait partie de l'enseignement cela devient, naturellement et sans discussion, obligatoire et c'est une démarche laïque. De même les confusions des enseignants sont graves quand ils mettent sur le même plan des documents aussi différents que les Évangiles et la Guerre du Péloponnèse de Thucydide. Les documents doivent être abordés dans un même esprit rationnel, mais en sachant qu'ils ne sont pas du même ordre et qu'il ne renvoie pas à un même régime de vérité. 
Mark Sherringham: Je suis tout à fait d'accord sur la nécessité de cette réflexion de nature philosophique qu'il faudrait proposer aux futurs enseignants sur le statut de la vérité, sur les différents régimes de vérité, et sur le rapport du religieux aux autres types de discours. Du point de vue des futurs enseignants, c'est lorsqu'ils sont dans un contexte d'adaptation à des publics variés, hétérogènes ou qui posent tout à coup des difficultés que la question est soulevée. Nous allons être confrontés de plus en plus, dans les IUFM, au problème du religieux chez nos étudiants et professeurs stagiaires. C'est encore marginal aujourd'hui mais de plus en plus de jeunes femmes candidates pour devenir professeurs vont arriver voilées. Les IUFM ne sont pas concernés par la loi puisqu'ils n'accueillent pas des élèves mais des étudiants ou des professeurs stagiaires. En ce qui concerne ces derniers, c'est simple: la neutralité du fonctionnaire est exigée par son statut. C'est ce qui s'applique en deuxième année d'IUFM. La difficulté, sur le plan juridique, concerne les étudiants de première année qui sont étudiants et non professeurs stagiaires. Dans la mesure où ils se destinent quand même à un parcours professionnel bien ciblé, que peut-on faire?

André Legrand: Tant qu'ils ne sont qu'étudiants, la liberté est la règle. La coupure se fait quand on devient fonctionnaire, même stagiaire. Ce qui m'a beaucoup frappé dans la loi récente, c'est qu'à la suite de l'amendement parlementaire qui y a été intégré, elle est très largement devenue une loi de procédure où ce que ce qui est important, c'est la nécessité d'agir de telle façon, de négocier, etc. C'est totalement contraire à la tradition française et cela va poser le problème du rapport entre la norme et la procédure. Les personnels réclamaient un texte supposé tout résoudre. À partir du moment où il n'a pas été possible de le leur donner, compte tenu des contraintes internationales, et où l'on a défini une attitude à adopter, on a changé de dimension dans l'organisation et dans la régulation. Tout cela est inséparable de la relation enseignant/enseigné et du problème du système d'autorité à l'intérieur de l'école. On sait bien que le système traditionnel d'autorité de droit divin est, partout, en train d'exploser. Et on voit bien quels problèmes cela pose aux enseignants qui ont des réactions de crispation, mais qui sont cependant bien obligés de gérer cette question.

André Blandin: Nous sommes affrontés à une vraie difficulté : où sont les lieux et les possibilités d'une parole d'autorité? D'autre part, les jeunes associent trop facilement sincérité et vérité. Enfin, comme disait Bernard Toulemonde lorsqu'il préparait les programmes d'ECJS ${ }^{4}$, "on ne joue pas à la démocratie dans l'école, on la prépare». La relation est au cœur de l'école, mais elle est dissymétrique, les statuts différents doivent être respectés.

4. ECJS : éducation civique, juridique et sociale. 
Philippe Le Guillou: Il y a un lien très fort entre l'autorité intellectuelle et l'autorité pédagogique surtout lorsqu'il s'agit de prendre en compte le religieux. C'est vrai qu'il faut tout autant renoncer à une dimension catéchistique ou apologétique qu'éviter de désacraliser ces contenus. Dans certains manuels de la classe de sixième, on invite les élèves à lire le récit de la chute dans la Genèse en n'en considérant que le schéma narratif. Je crois que c'est là le résultat d'une certaine "rigidification» d'ordre techniciste dont la discipline des lettres a été victime pendant toute une période, ainsi que de la relation ambiguë, difficile, qu'elle entretient avec la question du sens. Il est peut-être plus simple de regarder des morphèmes, des constituants, la syntaxe et la grammaire du récit que d'aller s'aventurer dans des eaux plus troubles voire plus dangereuses. Ce risque de désacralisation me paraît donc tout à fait important. Il y a un juste milieu à inventer, et ce n'est pas simple, entre une matière qui est vivante et un objet de croyance pour certains et quelque chose qui est également de l'ordre d'une représentation, d'une écriture, d'une transformation, d'une configuration et a sa place, à ce titre, dans l'esthétique et pas seulement dans la croyance.

Dominique Borne: La comparaison éclairante est celle de l'approche de l'œuvre d'art. Le religieux, comme l'art utilise la démarche symbolique. On n'épuise pas le sens d'une ouvre d'art et on peut craindre les abus de l'acharnement didactique à vouloir tout expliquer. À vouloir tout expliquer, tout dire, rendre tout transparent, on tue l'œuvre, comme on risque, dans le domaine du religieux de tuer le sens. L'approche doit être rationnelle mais elle ne doit pas détruire l'objet lui-même. Je vous propose maintenant de distinguer l'établissement et la classe. André Blandin a expliqué ce qu'est le caractère propre pour l'enseignement privé: un inspecteur public inspectant une classe du privé doit y trouver la même chose que dans une école du public. C'est un principe fondamental. Si on est dans la même situation que dans le public, pourquoi la loi sur le voile distingue-t-elle le public et le privé?

André Legrand: On est en train de tourner autour de la vraie question. Pourquoi le problème se pose-t-il maintenant? Il y a eu une sorte de révolution. On interprétait les grands textes fondateurs de l'école laïque en disant que le religieux, étant en dehors de la sphère publique, relevait d'un autre monde. Pas plus tard qu'hier, mes étudiants en sciences de l'éducation ne comprenaient pas les problèmes juridiques liés aux dispositions de la loi de 1905 sur les aumôneries. Ils trouvaient tout à fait logique la présence d'aumôneries dans les prisons ou dans les casernes mais la refusaient dans les écoles. J’ai dû leur rappeler que certains élèves étaient en internat et qu'à l'école comme partout, la religion devait aller à ceux qui n'avaient pas la possibilité d'aller à elle. C'est d'ailleurs la preuve que cette loi était aussi une loi de liberté religieuse puisqu'elle donnait les moyens d'une pratique religieuse à ceux qui le souhaitaient. 
Dominique Borne: Il suffit que des familles le demandent pour qu'il y ait des aumôneries.

André Legrand: Si l'on s'est heurté à des problèmes nouveaux, c'est précisément parce qu'on a admis chez les élèves cette possibilité d'exprimer leur opinion religieuse. L'aumônerie, c'était une possibilité collective qui concernait tout l'établissement. Dans la classe, la seule forme de liberté compatible avec la notion de neutralité de l'acte d'enseignement et de respect des convictions et des croyances de tous les élèves, ou avec la notion d'égalité que vous avez posée, c'est l'expression individuelle. Dès qu'elle acquiert une dimension collective, elle devient censurable.

Dominique Borne: Ne faudrait-il donc pas créer des espaces d'expression, y compris religieux, pour les élèves dans les établissements? C’est une question qu'on n'ose pas aborder actuellement.

André Legrand: Les aumôneries, c'était cela, non?

André Blandin: Le directeur d'un collège de Marseille qui reçoit $80 \%$ d'élèves musulmans et dans lequel il y a quelques cas de foulards islamiques me disait récemment: "nous sommes très stricts sur l'assistance à tous les cours et, en ce qui concerne le foulard, le dialogue est privilégié. Au bout de quelques semaines les jeunes filles acceptent de le poser». Le problème est résolu de façon pragmatique, mais pour combien de temps? La circulaire d'application de la loi du 15 mars insiste sur le dialogue. Être exemptés du champ d'application de la loi ne nous dispense pas de travailler à la construction du «vivre ensemble» et d'une intégration citoyenne, Paul Malartre, secrétaire général de l'Enseignement catholique l'a répété récemment. Revenons donc à la question que posait Alain Touraine en 1997 dans Comment pourrons-nous vivre ensemble, égaux et différents? Il demandait, je le cite de mémoire, de «refuser deux solutions également destructrices: une laïcité éliminant les différences et un communautarisme où les communautés seraient liées par la violence et le marché». Comment en sortir? Est-ce que je rencontre l'autre uniquement en ce qu'il me ressemble ou est-ce que son altérité, dit aussi quelque chose de ma propre identité et construit l'humanité? Le but de l'École est aussi celui-là : comment partir des différences pour construire ensemble une identité commune, un «vivre ensemble»?

Dominique Borne: Bien entendu. L'autre est respectable, non pas parce qu'il est différent mais parce qu'il est le même. Une question: dans les établissements catholiques où il y a des musulmans, est-ce qu'on parle de l'islam et comment?

André Blandin: Nous avons les mêmes difficultés d'inculture religieuse, parmi d'autres formes d'incultures, que dans l'enseignement public. Nous constatons les mêmes déficiences et avons le même travail à faire en termes et 
de formation des enseignants à la transmission de ces connaissances. Mais une question demeure: sommes-nous exactement dans la même situation que l'enseignement public? Sommes-nous dans une extériorité «objective » ou aussi, au-delà de la connaissance, dans une reconnaissance des autres religions?

Dominique Borne: Vous risquez d'avoir à accueillir, dans l'enseignement catholique, les élèves rejetées de l'enseignement public? Y avez-vous réfléchi? C'est un problème très concret.

André Blandin: Pour l'instant, il n'y a pas d'indices significatifs, mais votre question est bien réelle! Quand des parents musulmans viennent en disant: "au moins, chez vous, ils entendront parler de Dieu », qu'est-ce que cela veut dire? Est-ce que cela renvoie au désir d'une parole d'autorité? À une transmission de valeurs, au souhait d'un refuge contre un athéisme ambiant, un type d'éducation?

Dominique Borne: Pour aller dans le même sens, je me souviens d'un débat public au cours duquel un père d'élève se félicitait du travail de Régis Debray parce qu'enfin on allait introduire la morale à l'école grâce à la religion. Cette lecture-là est dangereuse. Jean-Paul Willaime, on estime la population musulmane religieuse pratiquante en France à $10 \%$ ou $12 \%$. Sait-on si, dans le cas des élèves musulmans qui manifestent des signes - ramadan, voile, etc. -, c'est réellement religieux? On a tendance, actuellement, à rejeter tous les enfants de l'immigration du côté de la religion musulmane. Comment le sociologue voit-il ce problème-là?

Jean-Paul Willaime: Il faut tenir compte du fait qu'il est toujours difficile, y compris en sociologie dont ce n'est d'ailleurs pas le rôle, de mesurer l'authenticité des engagements religieux. On dispose d'indicateurs concernant des pratiques, des gestes, des coutumes etc. mais on rencontre aussi bien des «musulmans du ramadan » que des « juifs de Kippour » et des "chrétiens de Noël », c'est-à-dire des personnes qui n'ont plus qu'un lien très lâche avec leur tradition religieuse... Quant à la statistique religieuse, elle est confrontée à un gros problème à cause d'un principe adopté au nom de la laïcité, à savoir qu'il est illégitime, dans les recensements nationaux, de demander l'appartenance religieuse des Français même sous couvert d'anonymat. Ce qui fait que pour les statistiques concernant l'islam, comme le bouddhisme d'ailleurs, on obtient des fourchettes énormes. C'est vrai que considérer le nombre de musulmans à partir de patronymes, de prénoms, d'origines géographiques ou ethniques est parfaitement illégitime. Il faut peut-être réviser une certaine représentation spontanée du religieux que nous avons tous en tant que Français. Nous avons tous peu ou prou intériorisé un principe selon lequel la religion, c'est le for intérieur, le privé, l’individuel. Or les historiens, les sociologues, les spécialistes des civilisations savent bien que c'est un fait collectif et un fait social. L'approche inter- 
disciplinaire du fait religieux doit nous faire redécouvrir les différentes dimensions du religieux contre l'approche restrictive du fait religieux qui s'est développée, au nom de la laïcité et dans un certain contexte. Une approche qui réduirait le religieux au for intérieur et au spirituel, qui l'identifierait purement et simplement à l'irrationnel et à la soumission au pouvoir d'un clerc, n'est du reste pas très laïque car elle méconnaît la nature socio-culturelle du religieux, son caractère de structure symbolique qui dépasse les aléas des pratiques et des représentations des individus.

Dominique Borne: Ceux qui observent le ramadan vont-ils à la mosquée?

Jean-Paul Willaime : Non, loin de là. Cela nous rappelle qu'une religion, c'est aussi une identification à un monde symbolique qui se fait parfois de façon implicite, confuse et pas toujours bien informée. C'est une façon de se définir comme être humain.

Dominique Borne: Constatez-vous une différence de générations dans le monde musulman? Y a-t-il une manière de manifester du religieux différente dans les générations des jeunes adultes par rapport à la génération de leurs parents?

Jean-Paul Willaime: D'après ce que je connais des travaux de collègues travaillant notamment sur les populations de jeunes musulmans, dans le cadre d'un retour plus général des affirmations identitaires qui dépasse le domaine religieux, on constate aussi dans les jeunes générations un besoin de s'affirmer, d'afficher une identité religieuse. Mais cette dernière n'est pas exclusive d'autres identités. Le travail de Nicola Tietze, docteur en sociologie de l'EHESS, sur les jeunes musulmans en France et en Allemagne montre que ces jeunes femmes et ces jeunes hommes s'identifient comme musulmans tout en se sentant pleinement Français ou pleinement Allemands. Ils « travaillent » leur identité de musulmans, ils en jouent prenant certains éléments, en rejetant d'autres, c'est pour eux une façon de s'inscrire dans l'espace français ou dans l'espace allemand tout en disant leur différence. L'identification à la religion musulmane leur permet de dire que, tout en étant Français, ils ne sont pas tout à fait comme les Français d'origine auvergnate ou bretonne. C'est leur façon de décliner leur identité plurielle.

Dominique Borne: C'est un point tout à fait important et intéressant. Pensez-vous que si l'islam était plus visible en France, s'il y avait davantage de mosquées, il aurait moins besoin d'affirmation, y compris dans l'école?

Jean-Paul Willaime: Le renversement de la stigmatisation est un processus social bien connu. Plus on est stigmatisé, plus on a tendance à afficher son identité. Des personnes s'affirmeront d'autant plus comme musulmanes que la religion musulmane sera socialement peu visible et culturellement 
peu acceptée. Le problème de la visibilité, de la publicisation des identités religieuses se pose différemment selon les mondes religieux. Chez les catholiques et les protestants - c'est un peu moins net chez les orthodoxes -, on peut dire qu'il y a eu une tendance à l'invisibilisation, à une baisse de la publicisation de l'identité religieuse. En Alsace, selon qu'on était catholique ou protestant, y compris au niveau de la couleur de la coiffe, il y avait une visibilisation sociale de l'identité confessionnelle. Or tout cela a été privatisé, intimisé et devient du coup incognito. Par contre, avec le judaïsme et avec l'islam, la question de la visibilité se pose différemment, d'autant plus que dans le cas de l'islam, une partie importante des personnes s'identifiant à cette religion ont des origines géographiques diverses, d'autres coutumes vestimentaires, etc., ce qui contribue à accentuer cette visibilité.

Dominique Borne: La loi Debré pourrait-elle permettre d'ouvrir des écoles privées musulmanes?

André Legrand: Normalement oui, mais en en respectant les conditions.

Dominique Borne: En ce qui concerne les départements toujours sous régime concordataire, les musulmans sont-ils fondés, légalement, à réclamer un enseignement officiel de l'islam dans un établissement public?

André Legrand: L'exemple allemand montre la difficulté des questions que cela soulèverait. L'organisation allemande est fondée, grosso modo, sur un armistice catholique/protestant et les dispositions applicables sont, pour l'essentiel, des articles qui viennent de la Constitution de Weimar et qui ont été repris par la Constitution de Bonn. L'irruption d'une religion nouvelle est perturbante. La plupart des auteurs indiquent qu'il n'y a pas de raison juridique pour qu'un enseignement de l'islam ne puisse pas se faire. Mais, en même temps, il faut bien constater qu'il ne se fait pas.

Mark Sherringham: Oui, il n'y a pas de raison autre qu'historique et d'autre part, il y a aussi un problème d'organisation: qui prendrait cela en charge?

André Legrand: En Allemagne, le problème est le même. On sait qui représente l'Église évangélique d'Allemagne, ou l'Église catholique mais en ce qui concerne les musulmans, c'est beaucoup plus compliqué à déterminer. Or, en droit allemand, ce n'est pas l'État qui a la responsabilité de l'enseignement religieux; ce sont les Églises qui, à l'intérieur du système scolaire, le prennent en charge.

André Blandin: Lorsque nous parlons du fait religieux s'agit-il d'un concept isolé, d'une réalité passée, d'un patrimoine à transmettre ou n'est-ce pas plutôt dans l'ordre de la vie, du développement, de la confrontation et du 
dialogue? L'École est traversée par les problèmes de la société de son temps, mais elle ne peut être simplement à sa remorque, elle est en même temps un lieu de résistance, elle est porteuse de valeurs et d'un projet pour l'homme.

Dominique Borne: Il faudrait d'ailleurs comparer, de ce point de vue, les écoles privées juives et les écoles privées catholiques qui sont très différentes. Une donnée objective pour le montrer: l'enseignement de l'hébreu est, en France, presque exclusivement assuré par les écoles privées juives sous contrat. Il n'y a pratiquement pas d'enseignement de l'hébreu dans l'enseignement public. On peut penser qu'il s'agit là d'un phénomène communautaire fort.

André Legrand: C'est là que la loi Debré est contraignante: tous les enfants, sans distinction d'origine, d'opinion ou de croyance ont accès aux établissements privés sous contrat. Certes, les conditions d'ouverture sont limitées par les critères de police, moralité, sécurité et salubrité mais l'État a effectivement, avec cette loi, sauvegardé ses droits et ses intérêts.

\section{Conclusion}

Mark Sherringham: Je crois qu'une étape a été franchie dans le domaine de la formation des enseignants mais que la réflexion doit se poursuivre. En pratique, les IUFM n'ont souvent pas encore vraiment mis en place la formation sur le religieux ou ont des difficultés à le faire. Il y a bien deux problèmes différents. L'un porte sur la façon d'intégrer le religieux, de l'enseigner dans le cadre de sa discipline. C'est un problème de connaissance et de relation de savoir. Mais il faut aussi savoir faire face, quelle que soit sa discipline, à l'irruption du religieux dans la classe, ce qui est un problème différent et très compliqué. Je rejoins ici ce que disait Dominique Borne: s'il n'est pas certain que la formation doive nécessairement lier philosophie de la laïcité et histoire du fait religieux, les deux doivent cependant avoir leur place dans la formation des enseignants.

André Blandin: Je m'arrêterai sur ce qui a été dit tout à l'heure sur le fait religieux: s'agit-il d'une chose morte qu'on dissèque comme un patrimoine qu'on se transmet ou est-ce encore dans l'ordre de la vie, du développement, de la confrontation et du dialogue? L'École et la société sont confrontées à cette question. L'École est à la remorque de la société mais elle est en même temps porteuse de valeurs et porteuse d'un projet pour une société future.

Jean-Paul Willaime : L'enseignement du fait religieux à l'école publique laïque représente un immense défi. Il ne faudrait pas non plus tomber dans une approche positiviste qui serait à la remorque de la dernière découverte archéologique ou épigraphique et oublier que les faits religieux sont, dans le passé comme dans le présent, des mondes convictionnels qui mobilisent fortement des hommes et des femmes, des langages symboliques à travers lesquels des 
hommes et des femmes disent la condition humaine. La façon dont les hommes disent la divinité, le sens de la vie et de la mort, du bonheur et du malheur, tout cela est toujours en même temps une façon de voir le monde et de concevoir la vie individuelle et collective. C'est donc un fait social et culturel aux multiples dimensions dont les œuvres et les textes sont les traces. C'est ce matériau que nous avons à comprendre et à analyser, en joignant la forme au sens.

Philippe Le Guillou: Je crois qu'il faut effectivement dialoguer, mais on ne saurait tout admettre. Lorsqu'on est à l'école, on souscrit à un certain nombre de règles. Avoir un enseignement à la carte, retenir certaines disciplines, en éviter ou en récuser d'autres, choisir ce qui va dans le sens de ses convictions philosophiques, religieuses, politiques est tout à fait impensable. Cette ligne de partage entre ce qui est de l'ordre d'un patrimoine et ce qui relève d'une croyance vitale, existentielle est très importante. En ce qui concerne l'enseignement des lettres, on n'aborde pas Agrippa d'Aubigné comme on aborde Voltaire et on ne peut pas lire Baudelaire comme on lit Bernanos. Quelle était la place du religieux dans la quête spirituelle, personnelle, esthétique de ces artistes dont le champ d'interrogation était extrêmement riche et large? C'est là-dessus qu'il faut insister aujourd'hui dans le cadre d'une sanctuarisation à mon sens absolue de la classe et de la neutralité de l'acte pédagogique.

André Legrand: Les pays européens se rapprochent; partout, on observe un mouvement, lent mais assez général, vers la sécularisation. Il reste quand même des différences considérables et on fait constamment l'expérience de la difficulté que l'on éprouve à expliquer à des publics étrangers ce qu'est la laïcité française.

Dominique Borne: Ma conclusion sera en forme d'écart. N'abuse-t-on pas aujourd'hui des identités religieuses? Il y a d'autres mémoires qu'il faudrait revivifier; elles aussi portent sens. Il y a une mémoire du travail, une mémoire des travaux des champs, de l'usine, de la mine, une mémoire des luttes syndicales. Mais il y a aussi une mémoire de l'immigration et des luttes coloniales. Toutes ces mémoires, l'histoire doit leur faire place et leur donner, raisonnablement, sens. Ainsi, chacun trouve identité et couleur dans une communauté qui est d'abord celle des citoyens. 